\title{
Direccionamiento y Evaluación del Trabajo Independiente del Estudiante en un Curso de Matemáticas Básicas
}

\author{
Juan C. Molina ${ }^{1}$
}

\section{Resumen}

El presente artículo, da cuenta sobre la experiencia de utilizar el portafolio de evidencias de desempeño de los estudiantes (P.E.D), como una herramienta metodológica que busca mejorar los resultados en cuanto a la construcción de competencias y optimización del trabajo independiente (T.I) articulado al microcurrículo del curso de matemáticas básicas del INSTITUTO TECNOLÓGICO METROPOLITANO. La estrategia ideada en el marco del mejoramiento del pensamiento reflexivo y crítico de los estudiantes, plantea un modo de direccionamiento del T.I sobre el desarrollo de las dimensiones cognitiva, subjetiva y publicosocial de los estudiantes, como líneas generales sobre las cuales se inscriben los procesos de desarrollo de competencias. Las actividades diseñadas alrededor de la estrategia, se orientan a recopilar evidencias que, por su significado y estado de elaboración, permiten establecer avances en los indicadores de logros asociados a las competencias proyectadas en el curso. Como consecuencia de la experiencia, se resaltan algunos resultados relacionados con el aumento de la autoconfianza del estudiante, la apropiación reflexiva de los contenidos del curso y la motivación hacia el estudio del área. Todo esto con repercusiones en lo actitudinal como aspecto importante en todo proceso de formación.

1 Facultad de Ciencias, INSTITUTO TECNOLÓGICO METROPOLITANO, juanmolina@itm.edu.co

Fecha de recepción: 12 de Agosto de 2010

Fecha de aceptación: 19 de Noviembre de 2010 


\section{Palabras clave}

Aprendizaje, competencias, desempeños, prácticas reflexivas, trabajo independiente.

\section{Abstract}

This paper realizes the student's performance evidence experience using the portfolio (P:E:D) as a methodological tool which seeks to improve, within the students' reflective framework and critical thinking development, the results regarding the skills development and self- work (T.I) optimization articulated to the Basic Math micro-curriculum course at the INSTITUTO TECNOLÓGICO METROPOLITANO (I.T.M). The strategy poses an addressing way of T.I around the cognitive development, subjective and social public-students as broadly on which are inscribed the competence-building processes. The activities designed within the strategy framework, aimed at collecting evidence, which by its meaning and status of development, allow for progress in the performance indicators associated with the powers envisaged in the course. As an experience result, we will analyze data related with student's auto confidence, his course content reflexive appropriation and the signature study motivation, all this with repercussions in attitudinal way as a meaningful topic in every formation process.

\section{Keywords}

Appropriation, attitudinal, confidence, learning strategies, self-work, skills development. 


\section{INTRODUCCIÓN}

La palabra portafolio está importada del término anglosajón "Portafolio Assessment" que se podría traducir como "carpeta de evaluación", o "portafolio process" cuya traducción final correspondería a "carpeta de aprendizajes" (Barragán, 2005). En la actualidad, es común escuchar el término 'portafolio de servicios' como evidencia física o estrategia empresarial que demuestra las competencias y servicios que una organización o persona está en capacidad de ofrecer en el mundo de la productividad.

En nuestro medio, se reconoce la estrategia de trabajo a través del portafolio como una estructura que ayuda a los estudiantes a visualizar su papel protagónico en la tarea educativa. Dicha estrategia induce a los estudiantes a entrar en procesos propios de autoformación que reflejan los avances y las dificultades que se presentan en los ambientes y condiciones de apropiación del conocimiento. En esta perspectiva, y para el caso de la presente experiencia, la filosofía de trabajo que define el portafolio, persigue el objetivo de desarrollar la competencia reflexiva de los estudiantes a través del reconocimiento del sentido y significado de las evidencias que reflejan sus esfuerzos y resultados alrededor del trabajo direccionado por el docente. El portafolio de desempeño se convierte en un instrumento y en una estrategia de acreditación individual que da cuenta permanentemente del desarrollo personal y profesional de cada estudiante.

El portafolio de evidencias de desempeño es un instrumento de registro de avances en el proceso de aprendizaje, es una herramienta que privilegia la práctica reflexiva de la evaluación propia de todo proceso de formación, es por tanto, personal; cada estudiante en coordinación con su docente hace registro, monitoreo y confrontación acerca de sus logros. El portafolio se asume como un diario académico que proporciona indicios y evidencias de lo que ha hecho y ha dejado de hacer quien aprende; de sus logros y dificultades; allí se describen los compromisos académicos asumidos y sus diferentes niveles de desarrollo (Días, 2005).

La estrategia planteada permite valorar la construcción de competencias a través de desempeños específicos, para el caso del desarrollo del microcurrículo de matemáticas del ITM, las 
actividades diseñadas para favorecer el desarrollo de estrategias de aprendizaje, hacen referencia a la elaboración de talleres y consultas, informes de utilización de mediadores, ensayos, relatorías, mapas mentales, mapas conceptuales, informes de contextualización, materiales gráficos, audiovisuales o en medio magnético que evidencian participación en procesos de mejoramiento académico construidos desde los distintos niveles de comprensión logrados por los estudiantes (Beltrán, 2003). El portafolio también puede incluir correcciones de pruebas, análisis críticos, reflexiones, posturas, avances en el proyecto de vida personal y profesional, así como propuestas, sugerencias, inquietudes, dificultades y aportes generales a las estrategias de enseñanza-aprendizaje, entre otros.

Para valorar los alcances de la utilización de esta herramienta, en el presente artículo se presenta, en primer lugar, una fundamentación y un modelo específico de portafolio diseñado para el curso de matemáticas básicas $\mathrm{y}$, en segundo lugar, se muestran algunos resultados obtenidos tras su utilización. Se indican además, en la parte final, algunas potencialidades y repercusiones de la estrategia en el proceso de formación de los estudiantes.

\section{REFERENTE INSTITUCIONAL}

Este informe surge como fruto de la experiencia de utilizar el portafolio de evidencias de desempeño como una herramienta de direccionamiento y evaluación del trabajo independiente (T.I) de los estudiantes en la asignatura de matemáticas básicas que ofrece la facultad de ciencias en los programas de tecnología del INSTITUTO TECNOLÓGICO METROPOLITANO. En la búsqueda de una mayor efectividad en los procesos formativos de los estudiantes, se trabaja en la actualidad sobre un proyecto de didáctica que busca articular la reflexión pedagógica en torno a la identificación de estrategias metodológicas de enseñanza y aprendizaje de las matemáticas en el marco del modelo pedagógico institucional (Urrego \& Castaño, 1999). De acuerdo a estos planteamientos y con el propósito de esclarecer los aportes que deja esta experiencia como metodología de trabajo, se pretende 
exponer el modelo de portafolio diseñado para la asignatura, así como los resultados más significativos obtenidos tras su utilización.

El objetivo principal que anima la experiencia del portafolio, se centra en la intención de articular y direccionar el trabajo independiente de los estudiantes en torno al desarrollo de competencias desde posturas que favorecen la comprensión y la reflexividad sobre el contexto en el cual se inscribe el estudiante como sujeto en proceso de formación. Como soporte de la estructura de créditos académicos, esta metodología busca un seguimiento y acompañamiento sistemático del estudiante. (Colombia, 2003). De este seguimiento surgen referentes para la evaluación del desempeño y verificación del estado de alcances de logros de los estudiantes; lo anterior en la vía de pensar que la innovación en procesos de enseñanza y aprendizaje debe propiciar, a su vez, y de manera natural, innovaciones en el componente de evaluación (Urrego \& Castaño, 1999).

\section{ALCANCES Y PROPÓSITOS DEL USO DEL PORTAFOபO}

Cuando se plantea el uso del portafolio como respuesta a una necesidad de articulación de metodologías que permitan direccionar el trabajo independiente de los estudiantes, se pensó en una herramienta que, además de orientar el trabajo 'extraclase', articulara la apropiación de estrategias de aprendizaje alrededor de las competencias a desarrollar en el microcurrículo del curso de matemáticas básicas. De esta manera, a través de la implicación del estudiante reflejada en su responsabilidad y compromiso frente a los procesos, se dio prioridad a la recolección y valoración de evidencias de desempeño como un asunto central en la evaluación de los procesos de enseñanza y aprendizaje. La utilización de la estrategia ha sido una invitación a los estudiantes para promover su participación metódica a sistematizar sus prácticas y experiencias $\mathrm{y}$, de manera general, a mejorar sus condiciones académicas mediante el fortalecimiento de los procesos de comprensión y contextualización del conocimiento.

El uso del portafolio de desempeño como técnica de 
direccionamiento, seguimiento y evaluación del trabajo independiente de los estudiantes, permite trazar en firme los objetivos que se persiguen en su utilización como una estrategia metodológica que estimula el aprendizaje; objetivos que deben apuntar a evaluar tanto el proceso como el producto, a desarrollar destrezas colaborativas y a promover la capacidad de resolución de problemas en los estudiantes. De igual forma, del uso del portafolio se espera: motivar al estudiante hacia la reflexión sobre su propio aprendizaje participando en su proceso de evaluación, disponer de un medio que permita estructurar las tareas de aprendizaje en actividades obligatorias, optativas, propositivas y reflexivas entre otras. De otro lado, la utilización de la estrategia provee al docente la información necesaria para ajustar los contenidos del curso a las necesidades y capacidades de los estudiantes (Cordero, 2002).

\section{EL PORTAFOLIO COMO RECURSO METODOLÓGICO}

Como punto de partida para el desarrollo del curso de matemáticas se tomó el microcurrículo aprobado por la institución para ser desarrollado en 16 sesiones semanales a razón de 4 horas por semana, para un total de 64 horas de trabajo presencial. De acuerdo a esta proyección, el trabajo independiente (TI), entendido como el tiempo que deben dedicar los estudiantes a la asignatura por fuera de las horas propias de clase, se estimó en 128 horas correspondientes al doble de horas directas de trabajo presencial con estudiantes.

La estructura de competencias y contenidos presupuestados para el curso se pueden observar en la Tabla 1.

Sobre la mirada del portafolio como un eje transversal a partir del cual se favorecen los aprendizajes y se evidencian las competencias desarrolladas por los estudiantes, se hizo necesario pensar en la organización de contenidos y la selección de tareas en función de los niveles de desarrollo de competencias esperados, así como de los indicadores de logro a avaluar (Rodríguez, 2006). 
Tabla 1. Competencias e indicadores del curso de matemáticas básicas ITM

Competencia de la unidad académica especializada

Aplicar los conceptos básicos de la aritmética, el álgebra y la trigonometría

como herramienta analítica, en la modelación y solución de situaciones problema, en contextos específicos de la ciencia y la tecnología, relacionados con su quehacer profesional.

Competencias específicas

Indicadores de logro

Utilizar adecuadamente los conjuntos numéricos, sus operaciones y propiedades básicas para solucionar situaciones problema en diferentes contextos.

Utilizar adecuadamente las expresiones algebraicas, sus propiedades básicas y operaciones para resolver situaciones problema en distintos contextos

Identificar y utilizar adecuadamente las expresiones trigonométricas, sus operaciones y propiedades básicas, como modelos para resolver situaciones problema en distintos contextos
Resuelve expresiones aritméticas utilizando las propiedades y operaciones de los conjuntos numéricos.

En una situación específica:

Plantea expresiones aritméticas.

Resuelve la situación a partir de la o las expresiones aritméticas que la representan, utilizando las propiedades, operaciones y/o métodos desarrollados.

Resuelve expresiones algebraicas utilizando las propiedades y operaciones algebraicas. En una situación específica:

Plantea la o las expresiones algebraicas que representan la situación.

Resuelve la situación a partir de la o las expresiones algebraicas que la representan, utilizando las propiedades, operaciones y/o métodos desarrollados.

Resuelve y representa inecuaciones con o sin valor absoluto en una situación determinada, a partir de sus definiciones y propiedades.

Resuelve expresiones trigonométricas, utilizando las propiedades y operaciones trigonométricas.

En una situación específica:

Identifica la o las expresiones trigonométricas que representan la situación.

Resuelve la situación a partir de las expresiones trigonométricas que la representan, utilizando las propiedades, operaciones y/o métodos desarrollados. 
En este sentido, y para el caso de la experiencia, se tuvo en cuenta criterios relacionados con el diseño de tareas que encontraran su significado en la experiencia práctica del estudiante esperando que éstas fueran percibidas como relevantes a sus intereses. Se pensó en actividades que fomentaran un pensamiento complejo y crítico, y que además se ajustaran al contexto y a la duración de la asignatura en relación con los créditos asignados por la estructura curricular. Se buscó que las tareas fomentaran el trabajo individual y grupal, sin perder su relación con las competencias que se pretendían desarrollar, hecho que favoreció de manera directa el proceso de evaluación.

En este orden de ideas, se plantearon actividades que permitieran buscar un equilibrio entre las distintas fuentes de información de tal forma que se facilitara la observación desde distintos puntos de vista de un mismo aspecto en relación a la apropiación de contenidos y la efectividad de las estrategias de enseñanza y aprendizaje aplicadas. Bajo estas consideraciones se trabajó con base en productos elaborados alrededor de materiales incluidos en el portafolio como: reflexiones sobre el componente teleológico institucional misión, visión y principios institucionales. Identificación de responsabilidades y objetivos de los estudiantes frente a su proceso de aprendizaje, relatos reflexivos que describen filosofías personales, metas frente al proceso de formación, concepciones personales sobre estrategias de aprendizaje y metodologías individuales de trabajo. Se tuvo en cuenta para la valoración de los procesos, los productos o resultados de las pruebas de entrada, evidencias de la participación en programas de mejoramiento académico como asistencias a asesorías, cursos complementarios y descripciones de los resultados de estos procesos. De igual forma, se incluyó en el portafolio registros de asistencia y reflexiones sobre el desarrollo de las clases. Justificaciones ante eventuales inasistencias, planes de trabajo de recuperación, constancias sobre innovaciones obtenidas en el proceso de aprendizaje y la valoración de su efectividad. Evidencias de la ayuda brindada a otros compañeros, autoevaluación de actividades de aprendizaje, informes de 
revisiones bibliográficas y búsquedas de información por medios electrónicos.

Los resultados de pruebas, evaluaciones, trabajos y talleres, fueron de gran importancia en la medida en que se hacía énfasis en las correcciones y validación de resultados. De esta manera, el diseño de tareas formativas encuentra una correspondencia con los contenidos de la asignatura de matemáticas básicas, y con las competencias presupuestadas en el curso.

En lo metodológico se adoptó un formato de guía de trabajo que permitió orientar las actividades a la luz de unos objetivos y unos recursos concretos de trabajo, para esto, se hace una plena identificación de la competencia y se describen las diferentes actividades de aprendizaje para ser realizadas por los estudiantes. En el formato también se hacen explicitas cuales son las evidencias de aprendizaje que el estudiante debe registrar así como, un espacio para conclusiones que le permita al estudiante reflexionar sobre los aprendizajes significativos logrados y las dificultades encontradas. Como espacio de intercomunicación y articulación de la propuesta se diseñó un blog con la idea de organizar las guías de actividades y ofrecer un espacio interactivo de consulta y de recopilación de información para los estudiantes (Molina, 2008). Esta herramienta de apoyo se puede consultar en el sitio web http://guiasprecalculo.blogspot.com/

En el desarrollo del curso se les ofreció a los estudiantes la oportunidad de incluir en sus portafolios, actividades reflexivas que concretan tareas específicas, esto es, además del resultado final de la actividad, una reflexión que da cuenta de los indicios del proceso formativo que ha conllevado a su realización. De esta manera, el portafolio se convierte en un instrumento de autoreflexión continua, ya que su desarrollo permite establecer comparaciones y análisis sobre los cambios producidos en el proceso de aprendizaje de los estudiantes, lo que fortalece además el papel del estudiante como centro del proceso de formación.

En el portafolio se pretendió articular, no sólo las actividades propuestas por el docente, sino también las actividades desarrolladas por los estudiantes y elaboradas por su propia iniciativa a partir de su motivación y compromiso frente a su proceso educativo. En este sentido, y sobre la idea de llevar un 
orden cronológico con el desarrollo del curso, se plantearon diversas guías de actividades de ejercitación en matemática operativa, comprensión de conceptos, búsqueda de contextos de aplicación y utilización de mediadores institucionales, (ver Tabla 2). Cada guía se relaciona con unos desempeños específicos que son los que componen la competencia de la unidad académica especializada del curso. Tales desempeños específicos se denominan elementos de competencia (Tobón, 2005).

Tabla 2. Descripción de las guías de actividades de aprendizaje articuladas al P.E.D

\begin{tabular}{|c|c|c|}
\hline $\begin{array}{l}\text { Guía } \\
\text { de T.I. }\end{array}$ & Objetivo & Alcances de la guía \\
\hline 1 & $\begin{array}{l}\text { Promover el diálogo y el conocimiento } \\
\text { entre los compañeros del curso. }\end{array}$ & $\begin{array}{l}\text { Promoción de buenas } \\
\text { relaciones interpersonales a } \\
\text { través de una dinámica de } \\
\text { Integración. }\end{array}$ \\
\hline 2 & $\begin{array}{l}\text { Hacer una puesta en común de los } \\
\text { pensamientos, ideas y roles que asumen } \\
\text { los integrantes del grupo a través de } \\
\text { diálogos intersubjetivos que permiten } \\
\text { un reconocimiento del otro. }\end{array}$ & $\begin{array}{l}\text { Identificación de las actitudes } \\
\text { de los estudiantes frente a la } \\
\text { matemática con el propósito de } \\
\text { mejorar las condiciones } \\
\text { motivacionales. }\end{array}$ \\
\hline 3 & $\begin{array}{l}\text { Aplicar los conceptos básicos de la } \\
\text { aritmética como herramienta analítica } \\
\text { en la modelación y solución de } \\
\text { situaciones problema en contextos } \\
\text { específicos. }\end{array}$ & $\begin{array}{l}\text { Comprensión de las } \\
\text { propiedades y relaciones } \\
\text { aritméticas. }\end{array}$ \\
\hline 4 & $\begin{array}{l}\text { Conocer el componente teleológico } \\
\text { institucional como principio rector de la } \\
\text { propuesta educativa que ofrece el ITM. }\end{array}$ & $\begin{array}{l}\text { Identificación de aportes del } \\
\text { estudiante frente a la vivencia } \\
\text { de la Misión, Visión y } \\
\text { Principios institucionales. }\end{array}$ \\
\hline 5 & $\begin{array}{l}\text { Identificar los modos de acceso a la } \\
\text { información institucional así como a los } \\
\text { diferentes mediadores pedagógicos con } \\
\text { que cuenta el ITM }\end{array}$ & $\begin{array}{l}\text { Reconocimiento de espacios y } \\
\text { recursos institucionales: } \\
\text { Laboratorios, biblioteca y } \\
\text { página web institucional. }\end{array}$ \\
\hline 6 & $\begin{array}{l}\text { Resolver expresiones aritméticas } \\
\text { utilizando las propiedades y operaciones } \\
\text { de los conjuntos numéricos. }\end{array}$ & $\begin{array}{l}\text { Utilización del laboratorio de } \\
\text { matemáticas: Desarrollo de } \\
\text { laboratorio de } \\
\text { fraccionamientos. }\end{array}$ \\
\hline
\end{tabular}




\begin{tabular}{|c|c|c|}
\hline $\begin{array}{l}\text { Guía } \\
\text { de T.I. }\end{array}$ & Objetivo & Alcances de la guía \\
\hline 7 & $\begin{array}{l}\text { Practicar las operaciones básicas entre } \\
\text { polinomios y expresiones algebraicas. }\end{array}$ & $\begin{array}{l}\text { Comprensión de las } \\
\text { propiedades, relaciones y } \\
\text { operaciones con expresiones } \\
\text { algebraicas. }\end{array}$ \\
\hline 8 & $\begin{array}{l}\text { Definir, plantear, y resolver problemas, } \\
\text { como instrumento de reconocimiento de } \\
\text { estrategias y habilidades cognitivas } \\
\text { vinculadas con la búsqueda, selección, } \\
\text { adquisición, interpretación y } \\
\text { comunicación de la información. }\end{array}$ & $\begin{array}{l}\text { Identificación de estrategias de } \\
\text { aprendizaje en matemáticas. } \\
\text { Desarrollo de taller para } \\
\text { estudiantes. }\end{array}$ \\
\hline 9 & $\begin{array}{l}\text { Practicar los productos notables, } \\
\text { factorización y las operaciones básicas } \\
\text { entre fracciones algebraicas. }\end{array}$ & $\begin{array}{l}\text { Comprensión de los distintos } \\
\text { procedimientos de } \\
\text { factorización y operaciones con } \\
\text { fracciones algebraicas. }\end{array}$ \\
\hline 10 & $\begin{array}{l}\text { Simplificar expresiones en las que } \\
\text { aparecen exponentes positivos, } \\
\text { negativos y fraccionarios }\end{array}$ & $\begin{array}{l}\text { Comprensión de las } \\
\text { propiedades, relaciones y } \\
\text { operaciones con las potencias } \\
\text { de una expresión algebraica. } \\
\text { Manejo de exponentes en } \\
\text { general. }\end{array}$ \\
\hline 11 & $\begin{array}{l}\text { Sumar, multiplicar y dividir expresiones } \\
\text { donde aparecen expresiones radicales. }\end{array}$ & $\begin{array}{l}\text { Comprensión de las } \\
\text { propiedades, relaciones y } \\
\text { operaciones con expresiones } \\
\text { radicales. }\end{array}$ \\
\hline 12 & $\begin{array}{l}\text { Caracterizar las ecuaciones lineales, los } \\
\text { sistemas de ecuaciones lineales y las } \\
\text { ecuaciones cuadráticas. }\end{array}$ & $\begin{array}{l}\text { Solución de ecuaciones } \\
\text { lineales, cuadráticas y } \\
\text { sistemas de ecuaciones. }\end{array}$ \\
\hline 13 & $\begin{array}{l}\text { Discutir en grupos procesos para } \\
\text { plantear y solucionar problemas en } \\
\text { contexto donde se involucran } \\
\text { ecuaciones. }\end{array}$ & $\begin{array}{l}\text { Solución de problemas en } \\
\text { contexto que se resuelven } \\
\text { mediante ecuaciones. }\end{array}$ \\
\hline 14 & $\begin{array}{l}\text { Relacionar e identificar dentro del } \\
\text { espacio cotidiano, imágenes o } \\
\text { situaciones asociadas a contenidos } \\
\text { matemáticos }\end{array}$ & $\begin{array}{l}\text { Identificación de relaciones } \\
\text { matemáticas en el entorno a } \\
\text { través de la participación en el } \\
\text { concurso 'Toma tu propia foto } \\
\text { matemática'. }\end{array}$ \\
\hline 14.1 & $\begin{array}{l}\text { Identificar el Matlab como un software } \\
\text { de manejo de expresiones matemáticas } \\
\text { y graficación. }\end{array}$ & $\begin{array}{l}\text { Solución de ejercicios y } \\
\text { obtención de gráficas a través } \\
\text { del programa Matlab }\end{array}$ \\
\hline
\end{tabular}




\begin{tabular}{|c|c|c|}
\hline $\begin{array}{l}\text { Guía } \\
\text { de T.I. }\end{array}$ & Objetivo & Alcances de la guía \\
\hline 15 & $\begin{array}{l}\text { Visitar los distintos museos del } \\
\text { planetario y trabajar el material } \\
\text { audiovisual relativo a las ecuaciones } \\
\text { cuadráticas y aplicaciones de las } \\
\text { soluciones imaginarias y complejas. }\end{array}$ & $\begin{array}{l}\text { Identificación del planetario } \\
\text { municipal como un espacio de } \\
\text { divulgación científica de la } \\
\text { ciudad de Medellín adscrito al } \\
\text { I.T.M. }\end{array}$ \\
\hline 16 & $\begin{array}{l}\text { Realizar una prueba diagnóstica con el } \\
\text { fin de detectar en los estudiantes, el } \\
\text { estado de razonamiento y conocimiento } \\
\text { en matemáticas }\end{array}$ & $\begin{array}{l}\text { Revisión del cuestionario o } \\
\text { prueba de entrada trabajada } \\
\text { por el estudiante el primer día } \\
\text { de clase con el objetivo de } \\
\text { verificar los avances en los } \\
\text { conocimientos elaborados por } \\
\text { los estudiantes. }\end{array}$ \\
\hline 17 & $\begin{array}{l}\text { Obtener la descomposición en fracciones } \\
\text { parciales de una fracción propia } \\
\text { aplicando procedimientos de manejo de } \\
\text { expresiones algebraicas como } \\
\text { factorización y sistemas de ecuaciones. }\end{array}$ & $\begin{array}{l}\text { Utilización de procedimientos } \\
\text { de factorización y técnicas de } \\
\text { solución de sistemas de } \\
\text { ecuaciones para representar } \\
\text { una fracción como una suma de } \\
\text { fraccionas parciales. }\end{array}$ \\
\hline 18 & $\begin{array}{l}\text { Resolver ecuaciones y problemas } \\
\text { planteados utilizando las propiedades } \\
\text { de las relaciones exponenciales y } \\
\text { logarítmicas. }\end{array}$ & $\begin{array}{l}\text { Comprensión de las } \\
\text { propiedades y operaciones } \\
\text { donde aplican las relaciones } \\
\text { exponenciales y logarítmicas. } \\
\text { Solución de problemas. }\end{array}$ \\
\hline 19 & $\begin{array}{l}\text { Aplicar las técnicas y propiedades para } \\
\text { resolver inecuaciones. }\end{array}$ & $\begin{array}{l}\text { Solución de inecuaciones y } \\
\text { problemas aplicando las } \\
\text { propiedades de los números } \\
\text { reales y del valor absoluto. }\end{array}$ \\
\hline 20 & $\begin{array}{l}\text { Aplicar las relaciones trigonométricas } \\
\text { básicas }\end{array}$ & $\begin{array}{l}\text { Comprensión de las relaciones } \\
\text { trigonométricas y sus } \\
\text { aplicaciones }\end{array}$ \\
\hline 21 & $\begin{array}{l}\text { Evaluar la estrategia de trabajo } \\
\text { aplicada en el desarrollo del curso de } \\
\text { Matemáticas Básicas a través del } \\
\text { Portafolio de Evidencias de Desempeño } \\
\text { de los estudiantes. P.E.D. }\end{array}$ & $\begin{array}{l}\text { Evaluación por parte de los } \\
\text { estudiantes de la metodología } \\
\text { aplicada durante el semestre y } \\
\text { reconocimiento de logros } \\
\text { personales adquiridos. }\end{array}$ \\
\hline
\end{tabular}




\section{VALORACIÓN DEL PORTAFOLIO}

El portafolio como instrumento que evidencia la apropiación de aprendizajes, requiere de revisiones periódicas como parte de su seguimiento y retroalimentación; esto permite identificar la evolución de los estudiantes y establecer los niveles de desarrollo de competencias a través de la verificación de los indicadores de logro asociados a cada competencia. El proceso de revisión continua en esta experiencia, permitió compensar las necesidades de los estudiantes en algunos casos y, en otros, potencializar su proceso de formación, esto es, para el primer caso realizar actividades puntuales con los estudiantes que mostraban bajos niveles de desarrollo de competencias a fin de mejorar su desempeño y, en el segundo caso, plantear actividades de T.I que favorecieran el desarrollo de un nivel mayor de competencias en los estudiantes más avanzados del curso.

La evaluación del portafolio, entendida como una actividad de comprensión y valoración de los procesos y productos de los estudiantes, se soporta en la observación y contraste desde cierto grupo de indicadores preestablecidos y relacionados con la habilidad de organización y presentación de los resultados de las reflexiones y aprendizajes de los estudiantes. Algunos de estos criterios se agrupan en aspectos relacionados con: La presentación del Portafolio, redacción clara y comprensiva como evidencia del desarrollo de competencias comunicativas generales, resultados de actividades acordes al cronograma concertado con el docente y equilibrio entre la información aportada procedente de fuentes externas, del profesor, y la propia a fin de establecer avances en los niveles de creatividad y originalidad. Para la evaluación se tuvo en cuenta también la organización, capacidad de selección, integración y coherencia; evidencias de participación en actividades complementarias, aportes sobre temas y contenidos complementarios a lo desarrollado en clase, con ideas, sugerencias y actividades desarrolladas por iniciativa personal. De manera general se tiene en cuenta el grado de implicación, participación y compromiso asumido por el estudiante (Barragán, 2005). 


\section{RESULTADOS DE LA EXPERIENCIA}

En adelante se mostrarán algunos alcances de la experiencia retomados desde los resultados cuantitativos de los estudiantes de acuerdo a los parámetros institucionales $\mathrm{y}$, desde las percepciones de los estudiantes frente al impacto de la utilización del portafolio. Igualmente aparecen reflexiones por parte del docente implicado en la experiencia, como un insumo clave para el mejoramiento y mayor impacto en futuras implementaciones de la estrategia.

Los resultados del grupo de matemáticas básicas conformado por 42 estudiantes, 38 hombres y 4 mujeres, se pueden establecer de la Fig. 1. Se puede decir que el número de estudiante que aprueban -es decir el número de estudiantes que logran las competencias- es satisfactorio en relación a los resultados que en promedio registran los estudiantes de la misma asignatura en la institución. Esto induce a pensar, que la utilización del portafolio no ha tenido repercusiones negativas frente a los resultados académicos del grupo.

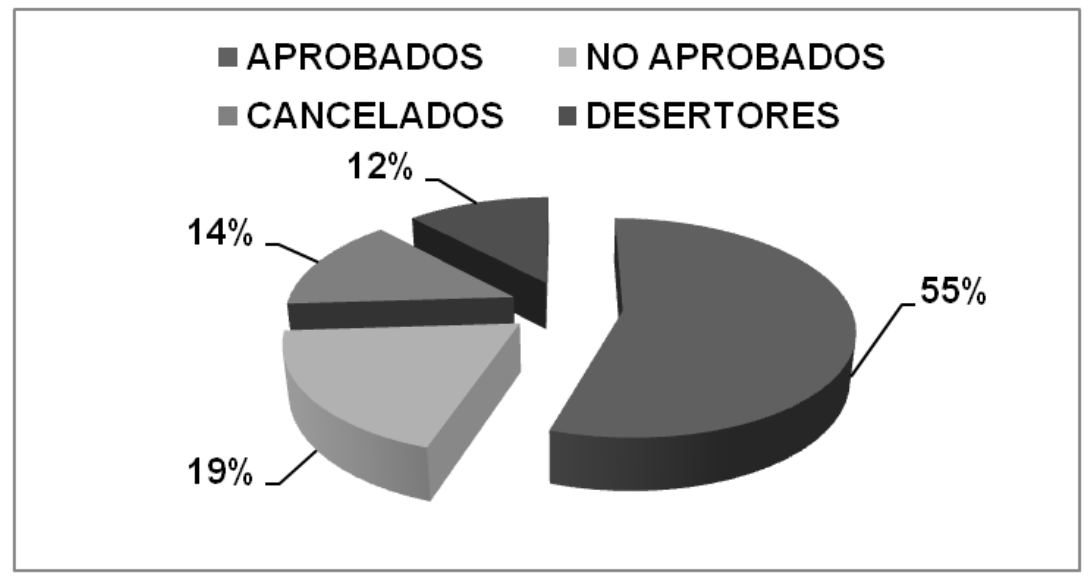

Fig. 1. Resultados del grupo de matemáticas básicas

Al considerar la situación del grupo en relación a los estudiantes que participaron de la totalidad del proceso, es decir 31 estudiantes que corresponden al total de matriculados menos los cancelados y los desertores, se puede establecer que el $74 \%$ 
logró las competencias, cifra que es significativa al considerar esta situación como un indicador de avance sobre la meta de aumentar el índice de estudiantes que logran las competencias por grupo, (ver Fig. 2).

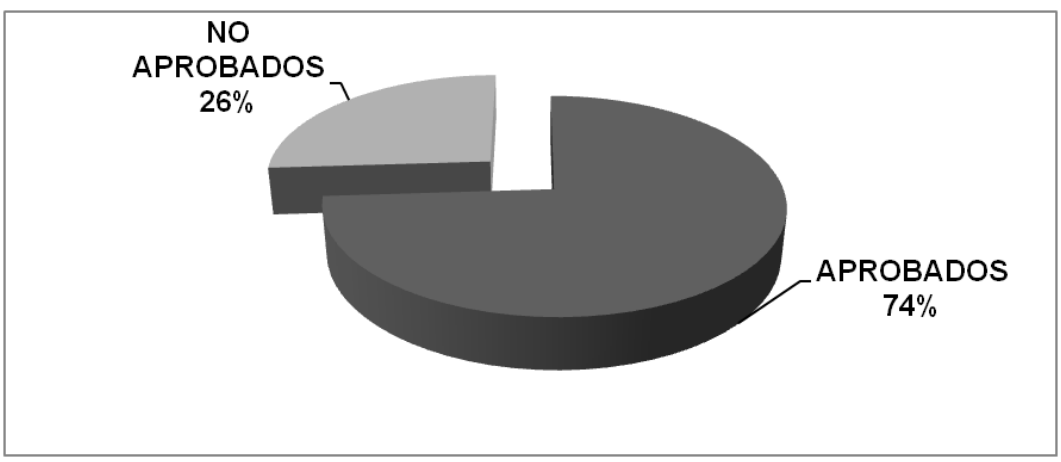

Fig. 2. Resultados de los estudiantes que culminaron el curso de matemáticas básicas

Es importante resaltar, que el aporte del portafolio en la evaluación definitiva del curso, se asocia con un peso porcentual del $30 \%$, ya que el $70 \%$ restante corresponde a una distribución evaluativa preestablecida en el microcurrículo con base en evaluaciones parciales y demás pruebas escritas de seguimiento. Sin embargo, como parte del proceso sustentado con el portafolio, se ha evidenciado que las actividades planeadas para el trabajo independiente, ejercen su influencia directa sobre los resultados de dichas pruebas escritas, ya que uno de los alcances esperados es justamente la preparación del estudiante para responder a este tipo de requerimientos. El portafolio permitió mayores niveles de competencia toda vez que los conceptos se abordaron desde diversas perspectivas y a través de actividades guiadas que permitían, dentro del TI, la ejercitación en torno a estrategias de aprendizaje válidas inclusive en el estudio de cualquier otra asignatura de las ciencias básicas.

El proceso de análisis merece también una mirada cualitativa, motivada por la confrontación natural que demanda el resultado preciso o numérico de los estudiantes, en relación a los indicadores planteados en la estructuración del portafolio. Esta información se 
sustenta sobre el contraste de la evaluación de las guías de T.I y de los testimonios de los estudiantes registrados en la última guía planteada, cuyo objetivo estaba orientado a identificar las percepciones del estudiante en cuanto al sentido, significado y aportes que se atribuyeron a la experiencia. En relación a estos planteamientos, se pueden establecer ciertas interpretaciones que dan cuenta de las elaboraciones de los estudiantes respecto a la conceptualización matemática y a las repercusiones de la utilización del portafolio en su formación como estudiantes. Aparecen entonces, indicios del aprovechamiento de la experiencia desde la perspectiva del estudiante y del docente, como soporte de nuevas miradas y configuración de sentidos entorno a la esencia e importancia de la participación en procesos formativos, esto es, se detecta un desarrollo de competencias que sobre pasa los propósitos iniciales en cuanto a las competencias académicas relativas a la matemática, trascendiendo a unos aprendizajes en los estudiantes que reflejan unas posiciones y conductas que garantizan una mayor asertividad en su formación, en lo que se podría denominar 'su formación para la vida'. A la pregunta ¿Que significó para usted el haber trabajado el curso a través del P.E.D?, aparecen testimonios que reflejan cambios de actitud y reconocimiento de elementos que potencian su quehacer en distintos contextos. Se evidencia cómo la experiencia se convirtió en una ayuda o apoyo para el aprendizaje que indujo además al estudiante a mantener un compromiso constante y responsable frente a la práctica y profundización de los conceptos $\mathrm{y}$ procedimientos trabajados. A continuación se relacionan algunas respuestas de los estudiantes frente a este cuestionamiento:

'Significo tener un apoyo de aprendizaje más completo y seguro, porque realizando cada actividad resolvíamos las dudas y cada tema nos quedaba más claro'.

'Fue mucha ayuda para mi aprendizaje ya que no sólo se aprende lo visto en clases si no que se refuerza de manera significativa y se aprovecha el tiempo libre en un buen aprendizaje'.

'Para mi el haber trabajado en el curso a través del portafolio de evidencia de desempeño fue una experiencia muy constructiva pues me ayuda a llevar un manejo del tiempo que le dedico al 
estudio y un orden a los trabajos de apoyo que realizan, me pareció una buena estrategia de aprendizaje pues pienso que a través de la practica se alcanzan grandes logros en un área tan compleja como lo es la matemática'.

'Fue un esfuerzo extra que tuve que realizar por mi horario fuera del ITM, pero me dio un gran sentido de responsabilidad $y$ una meta a superar'.

'Fue muy significativo para mi el hecho de haber trabajado con el portafolio ya que gracias a este practique mucho y aplique de una manera constante lo aprendido en curso. Además gracias a las dudas que me generaban algunos ejercicios me impulsaron a dirigirme a las asesorias para resolverlas'.

'Significo una ayuda, un mecanismo de apoyo para un proceso más completo'.

'Para mi significó mayor responsabilidad mayor honestidad me permitió ver mi relajo'.

Dentro de este análisis cualitativo, se puede también establecer que: la autoconfianza, el aprovechamiento del tiempo libre y la motivación hacia el esfuerzo, son unas de las características más relevantes que reflejan los estudiantes en los siguientes testimonios extraídos de las respuestas a la pregunta ¿Que aportes significativos cree que se pueden extraer de la utilización del portafolio como estrategia de direccionamiento y evaluación del curso de matemáticas básicas?

'Los aportes más significativos que deja la utilización del portafolio del curso de matemáticas son: La responsabilidad que adquiere el estudiante consigo mismo, desarrollo mental, un incremento en la producción intelectual, brinda una ayuda al estudiante para mejorar sus competencias'.

'Creo que todo el portafolio como tal es una muy buena estrategia de estudio de las matemáticas; porque va relacionado al buen aprovechamiento del tiempo libre ya que se repasa y refuerza lo visto en clases'.

'El (PED) direcciona de una excelente manera al estudiante, creándole incógnitas y haciéndolo esforzase para realizar los ejercicios'. 
[44] Direccionamiento y Evaluación del Trabajo Independiente del Estudiante en un Curso de Matemáticas Básicas

'Mejora la autoconfianza en el estudiante'.

'Tener cosas evidencias que muestran lo que está trabajando y al final ver dicho desempeño'.

$\mathrm{Al}$ indagar a los estudiantes por los aprendizajes significativos logrados a través de la experiencia de utilización del portafolio, surgen percepciones que van más allá de los contenidos del microcurrículo, de esta manera, se establecen valoraciones que, además de presuponer una apropiación de los contenidos del curso, enfatizan en aquellos aspectos que dan cuenta de avances en su formación integral y fijación de conductas o actitudes que permiten, desde la ética, un mejor desempeño en el mundo de la vida, en el mundo de las relaciones en lo individual, familiar y social. La siguiente agrupación de testimonios da cuenta de tales consideraciones:

'Las matemáticas no eran mi fuerte $y$ al entrar a esta institución me sentía muy inseguro pero a medida que fue pasando el tiempo y con la forma de estudio fui mejorando cada vez más'.

'Antes las matemáticas no me gustaban, pero con este curso descubrí que si se practican le voy cogiendo el lado bueno'.

'Control del tiempo que le dedico al estudio, pues se adquiere una responsabilidad académica que requiere dedicación'.

'Aprendí a ser más ordenado y a trabajar el día a día, ya que si uno se atrasa en los talleres se va llenando de trabajo'.

'Puntualidad, responsabilidad, amor por el estudio, respeto por los conocimientos ajenos, satisfacción'.

'Mejoré mi habilidad matemática, pues con la práctica constante resolvía las dudas que se me presentaban'.

'Estrategias de estudio. El orden es importante a la hora de estudiar, manejo de un programa tan útil como el Matlab, como plantear un problema para resolver la o las incógnitas, aprendí temas nuevos como factorización, Radicación, trigonometría, inecuaciones, fraccionarios, ecuaciones, aclaré dudas de los logaritmos y de la trigonometría'.

'Reconocimiento de la institución'.

'Utilización de las ayudas que me presta el ITM y los docentes, como las asesorías y las visitas al laboratorio'. 
'Utilizar textos como guías para resolver ejercicios'.

'Despertó la creatividad con la realización del trabajo con la actividad de fotografía matemática'.

'Siempre que uno tenga dificultades es bueno pedir ayudas para la materia, son excelentes las asesorías, y es bueno también visitar la biblioteca'.

'Aprendi a tener más compromiso conmigo mismo'.

'Ser una persona más segura de mi mismo'.

'A ser honesto en la realización de talleres y a buscar ayuda cuando era necesario'.

'Confianza para realizar las cosas que en algún momento creí no saber'.

El valor agregado de la utilización de la estrategia radica en que se favorece el desarrollo de habilidades reflexivas del estudiante, toda vez que, de acuerdo a los testimonios dados por estos, y confrontados en el momento de revisar sus elaboraciones, se evidencia un mayor compromiso del estudiante frente a su proceso de formación y frente al estudio de la matemática como una disciplina básica en el campo de la tecnología. El porcentaje de estudiantes que aprueban tiende a mejorar ya que, las guías de trabajo permiten al estudiante abordar los conceptos y aplicaciones de una manera sistemática y ordenada siempre en dirección al desarrollo de habilidades cognitivas que le permitan al estudiante mejorar sus estrategias de aprendizaje.

\section{CONCLUSIONES}

Los resultados mostrados justifican las bondades de la utilización del portafolio en la asignatura de matemáticas básicas desde el punto de vista de los estudiantes, sin embargo, la experiencia desde la perspectiva del docente, indica que el portafolio de evidencias de desempeño es una oportunidad para mejorar los procesos de comprensión y planificación de la práctica docente, permite además, la apertura de un espacio de reflexión sobre el alcance del compromiso y la responsabilidad del profesor frente a los retos pedagógicos, frente a la innovación, 
sistematización de experiencias y en general, frente a su papel como agente de cambio en el mejoramiento de los procesos de enseñanza. A manera de síntesis, el portafolio como parte importante de una propuesta de formación que hace énfasis sobre el trabajo del estudiante, se entendió como un orientador metodológico que permitió mantener en firme el horizonte de formación trazado, estar continuamente conectado con las metas presupuestadas y de manera particular, contar con un mecanismo que facilitó la lectura y confrontación diaria de los avances y dificultades en el camino de consolidar los logros esperados en los estudiantes.

\section{REFERENCIAS}

Barragán, R., (2005); El portafolio, metodología de evaluación y aprendizaje de cara al nuevo espacio Europeo de educación superior. Una experiencia práctica en la Universidad de Sevilla. En: Revista Latinoamericana de Tecnología Educativa. Vol. 4. No 1. Pp. 121-139

Beltrán, J., (2003); Estrategias de aprendizaje. En: Revista de Educación de España. Número 332. Septiembre-Diciembre. Pp. 55-73.

Colombia, (2003); Decreto 2566 de septiembre 10 de 2003; Por el cual se establecen las condiciones mínimas de calidad y demás requisitos para el ofrecimiento y desarrollo de programas académicos de educación superior y se dictan otras disposiciones. Diario oficial $\mathrm{N}^{\circ}$ 45308 de septiembre 12 de 2003.

Cordero, G., (2002); Consideraciones generales sobre el uso del portafolio de desempeño docente en educación superior. En: Revista Acción Pedagógica. Vol. 11. No. 2. Pp. 76-83

Díaz, J.J., (2005); El Portafolio de Desempeño: Una práctica reflexiva para lograr éxito en la formación y en el aprendizaje. 145p. Fondo Editorial Fundación Universitaria Luis Amigó, Medellín, Colombia.

Molina, J.C., (2008); Guías de trabajo para un curso de matemáticas básicas. Recuperado el 14 de mayo de 2010, de:

http://www.guiasprecalculo.blogspot.com/ 
Rodríguez, B.A., (2006); La didáctica basada en procesos, una alternativa para innovar las prácticas educativas en la educación superior. Recuperado el 15 de julio de 2009 de:

http://www.sadpro.ucv.ve/Ponencias/blanca-de-escontrela.pdf.

Tobón, S., (2004); Formación basada en competencias, pensamiento complejo, diseño curricular y didáctica. 258p. Ecoe Ediciones, Bogotá, Colombia.

Urrego, M.I., Castaño, L.E., (1999); Modelo Pedagógico Instituto Tecnológico Metropolitano, $2^{\mathrm{a}}$ Edición, 78p, Fondo Editorial ITM, Serie cuadernos de la escuela, Medellín, Colombia. 